\title{
APROXIMACIÓN A LA COOPERACIÓN EN INNOVACIÓN EN EMPRESAS DEL PROGRAMA DE ASOCIATIVIDAD Y DESARROLLO EMPRESARIAL SECTORIAL -PADES- EN ANTIOQUIA (COLOMBIA)*
}

\author{
ALEJANDRO CORONADO MEDINA**, ANDREA ECHEVERRI*** \& JOSÉ ENRIQUE ARIAS PÉREZ ${ }^{* * * * *}$ \\ UNIVERSIDAD DE ANTIOQUIA
}

Recibido/ Received/ Recebido: 08/04/2013 - Aceptado/ Accepted / Aprovado: 14/05/2014

\begin{abstract}
Resumen
El objetivo principal de este artículo es describir los aspectos más relevantes de las relaciones de cooperación en innovación entre empresas pertenecientes al Programa de Asociatividad y Desarrollo Empresarial Sectorial -PADES- de la Asociación Colombiana de Micro, Pequeñas y Medianas Empresas -ACOPI- en el departamento de Antioquia (Colombia). La investigación es de carácter exploratorio, se indagó fundamentalmente la opinión de gerentes / personal de la alta dirección y se utilizó el análisis descriptivo para establecer frecuencias en materia de actividades y resultados de innovación; agentes, motivos, obstáculos para la cooperación en innovación y fuentes de financiación. Los resultados muestran que las empresas, cooperan mayoritariamente con empresas del PADES, realizan innovaciones blandas, poco intensivas en conocimiento, sobre todo en materia organizacional y en marketing, financiadas mayormente con recursos propios. Además, que la falta de confianza y de cultura son sus mayores obstáculos para la cooperación en innovación.
\end{abstract}

Palabras clave: Cooperación en innovación, Gestión de la innovación, Capacidades de innovación.

\section{APPROACH TO COOPERATION IN INNOVATION OF COMPANIES FROM PROGRAMME OF PARTNERSHIP AND BUSINESS DEVELOPMENT SECTORAL -PADES- IN ANTIOQUIA (COLOMBIA)}

\begin{abstract}
The main aim of this article is to describe the most relevant aspects of cooperation relations in innovation between companies belonging to the Program of Partnership and Business Development Sectoral -PADES- of micro, small and medium companies Colombian Association -ACOPI- in Antioquia (Colombia). The research is exploratory in nature, and it is mainly focused in manager's views / senior management and descriptive analysis was used to establish frequency related to in-
\end{abstract}

* Este artículo de investigación es producto del trabajo de grado: Cooperación en innovación en empresas del Programa de Desarrollo Empresarial Sectorial -PRODES- en Antioquia, para la obtención del título de Economista en la Universidad de Antioquia.

** Economista, egresado de la Universidad de Antioquia. Correo electrónico: alejocm2012@gmail.com. Tel: 054 5361062. Dirección postal: Calle 19 A \# 52-17, Barrio Remansos, San Antonio de Pereira, Rionegro - Antioquia.

*** Economista, egresado de la Universidad de Antioquia. Correo electrónico: andreaeb10@gmail.com. Tel: 054 5361062. Dirección postal: Calle 80 \# 74-126 Apto. 201, Medellín.

${ }^{* * * *}$ Doctorando en Dirección de Empresas de la Universidad de Valencia; Magíster en Gestión de Ciencia, Tecnología e Innovación de la Universidad de Antioquia; Especialista en Gerencia de Sistemas y Tecnología, Universidad Pontificia Bolivariana; Administrador de Empresas, Fundación Universitaria Luis Amigó. Profesor del Departamento de Ciencias Administrativas, Universidad de Antioquia. Miembro del grupo de investigación Gestor, Universidad de Antioquia. Correo electrónico: jenrique.arias@udea.edu.co. Tel: (57) 4-2195846. Dirección: Calle 67 \# 53 - 108 of. 13-105. 
novation activities and results; agents, reasons, obstacles for cooperation in innovation and funding sources. The results show that companies mainly cooperate with PADES companies, they perform soft innovations with low intensity in knowledge especially in organizational and marketing matters financed mostly with own resources. In addition, lack of trust and culture are the biggest obstacles for innovation cooperation.

Keywords: Innovation cooperation, Innovation management, Innovation capabilities.

\title{
APROXIMAÇÃO À COOPERAÇÃO EM INOVAÇÃO EM EMPRESAS DO PROGRAMA DE ASSOCIATIVISMO E DESENVOLVIMENTO EMPRESARIAL SETORIAL - PADES - EM ANTIOQUIA (COLÔMBIA)
}

\section{Resumo}

\begin{abstract}
O objetivo principal deste artigo é descrever os aspectos mais relevantes das relações de cooperação em inovação entre empresas pertencentes ao Programa de Associativismo e Desenvolvimento Empresarial Setorial -PADES- da Associação Colombiana de Micro, Pequenas e Médias Empresas ACOPI- no departamento de Antioquia (Colômbia). A pesquisa é de caráter exploratório. Indagouse fundamentalmente a opinião de gerentes/pessoal de alta direção e se utilizou a análise descritiva para estabelecer frequências em matéria de atividades e resultados de inovação; agentes, motivos, obstáculos para a cooperação em inovação e fontes de financiamento. Os resultados mostram fundamentalmente que as empresas cooperam em sua maioria com empresas do PADES, realizam inovações brandas, pouco intensivas em conhecimento, sobretudo em matéria organizacional e em marketing, financiadas principalmente com recursos próprios. Além do mais, que a falta de confiança e de cultura são seus maiores obstáculos à cooperação em inovação.
\end{abstract}

Palavras chave: Cooperação em inovação, gestão da inovação, capacidades de inovação.

Coronado, A., Echeverri, A. \& Arias, J. (2014) Aproximación a la cooperación en innovación en empresas del Programa de Asociatividad y Desarrollo Empresarial Sectorial-PADES- en Antioquia (Colombia). En: Revista de la Facultad de Ciencias Económicas de la Universidad Militar Nueva Granada. rev.fac.cienc.econ, XXII (2).

JEL: O22, O31.

\section{Introducción}

La rapidez con que se producen los avances tecnológicos y el actual proceso de globalización hacen fundamental la habilidad de las empresas para explotar recursos y capacidades con el objetivo de innovar. En particular, las capacidades para generar, adquirir, adaptar y usar conocimiento son un factor crecientemente estratégico en la evolución de los niveles de competitividad de las organizaciones y constituyen un insumo esencial para las actividades de innovación de las micro, pequeñas y medianas empresas (Mipymes ${ }^{1}$ ), considerando su potencial para la flexibilidad estructural y su capacidad de adaptación, a la vez que es una cuestión de vital importancia, principalmente para las economías emergentes, dada su participación en el tejido empresarial de estos países.

En el caso de Colombia, las Mipymes de acuerdo a la clasificación de tamaño por número de emplea-

La ley 590 de 2000 (modificada por la ley 905 de 2004), establece que de acuerdo con la clasificación de tamaño por número de empleados la microempresa es aquella que tiene de 1 a 10 empleados, la pequeña empresa de 11 a 50 empleados, la mediana empresa de 51 a 200 empleados, y la grande empresa más de 200 empleados. 
dos, tienen una participación en el tejido empresarial del 99,9\% (DANE, 2005), de allí las microempresas conforman el $96,1 \%$ de los establecimientos, las pequeñas el $3,3 \%$ y las medianas el $0,5 \%$. Estos segmentos productivos generan la mayor cantidad de empleos, aproximadamente el 81\% (DANE, 2005), específicamente, las micro representan el $51,1 \%$ del empleo, las pequeñas el $17,4 \%$ y las medianas el $12,5 \%$. Por tanto, existe una creciente necesidad de formular e implementar estrategias de apoyo para las Mipymes, al ser un elemento clave en el desarrollo económico del país, por ser alternativas de generación de empleo, ingresos y activos para un gran número de personas.

De acuerdo a las características de este tipo de empresas les resulta muy difícil sobrevivir en un contexto de alta competencia, debido a factores como: una base limitada de recursos humanos y financieros, altos niveles de informalidad, bajos niveles de asociatividad, know how tecnológico, visión estratégica y planificación a largo plazo, así como limitado acceso a fuentes de financiación y falta de información acerca del entorno y el mercado (CONPES, 2007). En este contexto, las actividades formales de investigación, desarrollo e innovación $(\mathrm{I}+\mathrm{D}+\mathrm{i})$ pueden ser costosas y arriesgadas para las Mipymes, por lo que se ven obligadas a buscar mecanismos que permitan afrontar la competencia en el actual mercado globalizado, y mejorar las capacidades, recursos y flujos de conocimiento. Una forma viable para lograrlo es a través de la cooperación en actividades de innovación, la cual consiste en la "cooperación activa con otros agentes del Sistema de Innovación con el objetivo de realizar actividades innovadoras. Es decir, estas actividades consisten en acuerdos con otros agentes por los cuales las empresas comparten los costes y los beneficios de proyectos de innovación" (López, 2008).

Para diversos estudios entre las ventajas de cooperar en innovación se encuentran, la obtención de experiencia, la posibilidad de acceder a recursos externos mediante transferencia de conocimiento, el aprendizaje organizacional (Becker \& Dietz, 2004), el incremento del potencial innovador (Filiou \& Massini, 2009), la reducción de la incertidumbre y de los costos en los procesos de innovación, así como un incremento de las actividades de $\mathrm{I}+\mathrm{D}+\mathrm{i}$ y una mejora en las competencias al interior de la empresa (Alm \& McKelvey, 2000).

Particularmente, de acuerdo a varios estudios hechos para Colombia en cuanto a innovación en pequeñas y medianas empresas (Pymes), se destaca su pobre comportamiento innovador; en su gran mayoría, no logran fabricar productos con valor agregado como resultado de procesos formales de $\mathrm{I}+\mathrm{D}+\mathrm{i}$ (Giraldo et al., 2009), el impulso innovador proviene de la demanda, basándose en la imitación, impulsada por la copia y la adaptación (Malaver \& Vargas, 2006), además, se destaca un tradicional aislamiento en el que no existen fuertes vínculos con el exterior, por lo que las Pymes colombianas poseen límites de crecimiento intramuros que dificultan el acceso a tecnología, conocimientos y recursos financieros. Igualmente, para el caso antioqueño se detecta un sistema de innovación regional fragmentado, con una escasa conectividad entre los diferentes agentes (Cataño, Botero \& Vanegas, 2008).

En este contexto, se enmarca el presente artículo, el cual tiene como propósito principal describir los procesos de cooperación en innovación desarrollados por algunas empresas pertenecientes al Programa de Asociatividad y Desarrollo Empresarial Sectorial PADES- en Antioquia, de la Asociación Colombiana de Micro, Pequeñas y Medianas Empresas -ACOPI-. El PADES consiste en una estrategia de integración empresarial que tiene como base la asociatividad, con el objetivo de incrementar la productividad, la competitividad y la innovación, a través de la generación y el fortalecimiento de la confianza y la cooperación entre los participantes. De allí el interés y la importancia del estudio de éste grupo de empresas, debido a que fomentan la conformación de redes empresariales y aprendizajes colectivos y ayudan a cambiar el carácter individualista de los empresarios con el fin de lograr verdaderos encadenamientos productivos que permitan afrontar los retos de la economía globalizada.

El estudio tiene como propósitos adicionales reconocer los principales agentes con los cuales cooperan las empresas PADES, los obstáculos que encuentran para hacerlo e identificar los resultados obtenidos en 
materia de innovación, además, se recoge información acerca de los motivos que tienen las empresas para cooperar en innovación, la regularidad con que lo hacen y las fuentes de financiación utilizadas.

El artículo se organiza de la siguiente manera, se desarrolla en primer lugar el trasfondo teórico del estudio y una breve revisión de la literatura sobre la cooperación en innovación; luego se describen la metodología empleada para la obtención de la información y las limitaciones de esta; posteriormente, se explican los resultados empíricos; y por último, se presentan las conclusiones.

\section{Innovación}

La innovación es discutida en la literatura científica y técnica y se ha convertido en un emblema de la sociedad moderna. Esta se puede definir como la capacidad de generar e incorporar conocimientos para dar respuesta creativa a los problemas del presente (Jardón, 2011). Según el Manual de Oslo la innovación es: "la introducción de un nuevo, o significativamente mejorado, producto (bien o servicio), de un proceso, de un nuevo método de comercialización o de un nuevo método organizativo, en las prácticas internas de la empresa, la organización del lugar de trabajo o las relaciones exteriores" (OCDE, 2005).

Específicamente, este manual considera cuatro tipos de innovación dentro de la empresa: i) la innovación de producto, se refiere a mejoras en las características técnicas o funcionales, componentes, materiales, o la informática integrad; ii) la innovación de proceso, la cual implica la introducción de nuevos o mejorados procesos de producción y distribución; iii) la innovación de mercadotecnia consiste en aplicar nuevos métodos de comercialización que impliquen cambios significativos en el diseño o envasado de un producto, en su posicionamiento (nuevos canales de venta), promoción o tarificación; y iv) la innovación organizativa consiste en la introducción de nuevos métodos organizativos en las prácticas internas, encaminados a la reducción de los costos administrativos y de transacción para aumentar la productividad.

Inicialmente fue Schumpeter (1935), quien destacó la importancia del cambio tecnológico y la innova- ción como determinantes del crecimiento económico a través de la incorporación de conocimientos radicalmente nuevos, capaces de provocar cambios "revolucionarios" y transformaciones decisivas en la sociedad y la economía. Este autor considera la innovación como un resultado del esfuerzo individual e interno de las empresas u organizaciones. Define al empresario innovador como el capaz de causar inestabilidades en los mercados aplicando el conocimiento existente en forma de nuevas combinaciones. Sin embargo, durante los años setenta y ochenta, aparece la corriente evolucionista o neoschumpeteriana, en la cual la innovación y la tecnología se desarrollan y se difunden en un contexto de retroalimentación continua influenciada por características políticas, económicas, históricas e institucionales.

La aparición de esta nueva perspectiva trajo también nuevos planteamientos teóricos como el de la teoría de los sistemas de innovación, definidos como un conjunto de instituciones y actores sociales que, tanto por su acción individual como por sus interrelaciones, contribuyen a la creación, desarrollo y difusión de nuevas prácticas productivas. Desde este perspectiva, existe una visión de la innovación como un proceso social, y a la vez interactivo, enmarcado en un entorno especifico y sistémico; Lundvall (1992) propone entonces la idea de un sistema nacional de innovación (SNI) definiéndolo como "un sistema social dinámico, caracterizado por una retroalimentación positiva con una tendencia a su propia reproducción, donde la capacidad innovadora está determinada por la interacción virtuosa de los actores que conforman el sistema".

Dentro de la perspectiva del SNI se encuentran también los Sistemas Regionales de Innovación (SRI), los cuales reconocen la importancia del entorno regional de innovación para las pymes y otorgan un mayor peso a los acuerdos de cooperación empresarial, destacando que en particular, las micro, pequeñas, y medianas empresas hallan en los mecanismos de cooperación con otras empresas de su mismo tipo una estrategia para responder a las dinámicas actuales de la economía mundial (Albizu, Olazaran, Otero \& Lavía, 2011). Estos planteamientos refuerzan la importancia de la innovación como un proceso interactivo y acumulativo basado en los conocimientos 
tácitos localizados y las interacciones fundamentadas en la confianza.

\subsection{Cooperación en innovación}

El análisis económico sugiere la existencia de una serie de dificultades para alcanzar el nivel óptimo de las actividades innovadoras bajo competencia perfecta en los mercados. Como consecuencia de los altos niveles de incertidumbre asociados a los resultados de las actividades de innovación, la existencia de costos fijos altos, y costos hundidos, y el carácter del input "conocimiento" -libremente disponible una vez revelado- (López, 2008), las empresas asumen un riesgo importante en la realización de este tipo de actividades, y por tanto tienen pocos incentivos para la asignación de recursos en éstas, considerando, además, que las necesidades de financiación de determinadas actividades son cada vez más importantes.

Desde el punto de vista teórico existen varias aproximaciones que justifican la cooperación en innovación. Para la teoría de los costos de transacción, entendidos como de información, de negociación y de supervisión del cumplimiento de los contratos (Williamson, 1979), la empresa opta por cooperar como un camino intermedio entre acudir al mercado o hacer innovaciones individualmente (Boltri, 2006). Teniendo en cuenta que la forma organizacional más eficiente es aquella que minimiza no sólo los costos de producción, sino también los de transacción (Alm \& McKelvey, 2000), los costos de la innovación y la necesidad de un rápido ajuste ante los cambios tecnológicos, implican que las firmas necesitan compartir los costos y riesgos de sus actividades de investigación, desarrollo e innovación $(\mathrm{I}+\mathrm{D}+\mathrm{i})$ recurriendo a acuerdos, los cuales se basan en reglas de funcionamiento previamente acordadas de forma explícita o tácita.

Por lo tanto, las empresas en cada momento deben hacer un análisis de costo-beneficio para elegir la alternativa más adecuada en el mercado, internalizando los procesos o acuerdos de cooperación para gestionar procesos de innovación (Muñoz \& Montoro, 2006).
Desde una perspectiva dinámica, la cual tiene en cuenta además la incertidumbre de las dinámicas económicas, las alianzas entre empresas son exitosas si son de tipo vertical, es decir, entre firmas y proveedores, debido a que las alianzas entre competidores tienden a ser juegos de suma-cero. Destaca, además que las alianzas entre firmas en materia de innovación tienen éxito sólo cuando el desarrollo de nuevos productos es muy costoso o riesgoso para la empresa, en ese caso, resulta ser mejor alternativa cooperar para desarrollar innovaciones (Alm \& McKelvey, 2000).

Desde la teoría de la organización industrial (Schmalensee \& Willig, 1989; Tirole, 1988) la cooperación en innovación está determinada por la existencia de spillovers, la capacidad de absorción de conocimiento por parte de la empresa, el tamaño y la pertenencia a un grupo de empresas. Los spillovers son conocimientos fuente de las actividades de innovación que no se pueden apropiar, se presentan en flujos de entrada o de salida de información, los primeros se buscan maximizar a través de la cooperación y los segundos se buscan minimizar mediante alternativas de protección. En cuanto a la capacidad de absorción (Cohen \& Levinthal, 1989, 1990) se propone que el flujo de información y conocimiento, fruto de actividades internas de innovación o cooperación, es más útil cuando previamente se posee un stock de conocimiento que facilite la asimilación de la información. Por su parte, la pertenencia a un grupo de empresas otorgaría prestigio a éstas por lo que se vuelven más atractivas como socios potenciales.

Por otra parte, la teoría de recursos y capacidades (Wernerfelt, 1984; Barney, 1991; Barney \& Arikan, 2001), propone que las empresas de forma estratégica realizan un análisis de las ventajas de realizar una actividad internamente o a través de alianzas con otros agentes. La empresa, buscando mantener o mejorar su posición competitiva y un mayor poder de mercado, pretende acceder a nuevos recursos o capacidades que no pueden ser obtenidos en el mercado o internamente, sino mediante mecanismos de cooperación con otras firmas.

Además, existen otras aproximaciones teóricas, como la del enfoque estratégico (Porter, 1991; Powell 
\& Grodal, 2005), que considera la cooperación empresarial como una forma de mejorar la competitividad para luego incrementar beneficios y la teoría de la dependencia de recursos (Aldrich, 1976; Aldrich \& Pfeffer, 1976; Pfeffer \& Salancik, 1978), la cual hace referencia a la escasez de recursos como la explicación a la interdependencia entre organizaciones.

\subsection{Cooperación en innovación en Mipymes}

A pesar de que los procesos de innovación de las Mipymes suelen ser de carácter incremental (Albizu et al., 2011; Jaramillo, Lugones \& Salazar, 2001), con un predominio del conocimiento tácito (Albizu et al., 2011) y realización de actividades de investigación y desarrollo informales e internas (Chun \& Mun, 2010; Jaramillo et al., 2001), los mecanismos de cooperación de pymes constituyen un rasgo distintivo para su sobrevivencia y crecimiento (Pallares, 2000; Rojec \& Jaklič, 2002).

Las Mipymes encuentran a través de los procesos de cooperación en innovación con agentes externos una forma viable de aliviar sus desventajas relativas en materia de innovación, como la baja acumulación de conocimientos tecnológicos y el acceso limitado a recursos financieros, mediante el mejoramiento del flujo de conocimiento desde el exterior de la empresa, la complementariedad de esfuerzos de innovación internos (Chun \& Mun, 2010) y la explotación colectiva de oportunidades y habilidades en aquellas actividades de la cadena de valor en las que se encuentran involucradas (Rosales, 1997).

Adicionalmente, debido a que las Mipymes se encuentran más imbricadas en el territorio que las empresas más grandes, resulta fundamental la existencia de mecanismos en el entorno regional que puedan favorecer sus actividades innovadoras (Albizu et al., 2011; Lavía, Otero, Olazaran \& Albizu, 2011). Estos mecanismos abarcan aspectos institucionales, estructurales y de infraestructura del Sistema Regional de Innovación tales como el ambiente, las regulaciones institucionales, la trama de relaciones entre los agentes e instituciones y el funcionamiento macroeconómico (Jaramillo et al., 2001). En este contexto, se subraya la importancia de los valores compartidos, los canales de comunicación confiables y durade- ros (Jaramillo et al., 2001; Albizu et al., 2011) y la existencia de capacidades internas de las empresas como condiciones indispensables para que puedan participar en relaciones fructíferas de cooperación en innovación con agentes externos y mejorar sus posibilidades de integrar información relevante del exterior (Albizu et al., 2011).

A continuación, se presentan las variables claves identificadas en la literatura de la cooperación en innovación empresarial, las cuales sirven de base para la construcción del instrumento metodológico.

\subsection{Agentes y actividades para la cooperación en innovación}

La realización de actividades innovadoras depende en parte de la variedad y estructura de los vínculos de las empresas con las fuentes de información y de conocimiento. Cada vínculo conecta a la empresa innovadora con otros agentes como proveedores, clientes, competidores, otras empresas, universidades, grupos de investigación, centros de desarrollo tecnológico (CDT) u organismos de la administración pública (OCDE, 2005). Se destaca que los vínculos formales entre agentes de un sistema de innovación tienen un mayor impacto sobre la actividad innovadora de las empresas que los informales (Powell \& Grodal, 2005; Godoe, 2000; Jaklič et al., 2010).

Así pues, en materia de cooperación en innovación, las pymes, orientadas principalmente a los mercados regional y local, les otorgan gran importancia a las relaciones de cooperación con universidades, CDT y otras instituciones como la administración pública, gremios empresariales y cámaras de comercio. Por el contrario, las que tienen mayor participación en los mercados nacional e internacional, no suelen interactuar con agentes de conocimiento. Por tanto, el sistema de innovación regional presenta lo que la literatura especializada denomina fallos de redes, es decir, la falta de articulación y conexión entre actores económicos y sociales (Cataño et al., 2008).

En el contexto de las Mipymes de Antioquia, Cataño et al. (2008) señala que existe un sistema de innovación local que tiene más o menos desarrollado un 
esquema de agentes de innovación que interactúan de manera casi siempre informal y esporádica.

Algunos estudios (Cataño et al., 2008; Monroy, 2006) identifican los agentes más relevantes del entorno regional, destacando aquellas organizaciones e instituciones que inciden directamente en los procesos de innovación de las empresas objeto de estudio. Respecto a la cooperación empresa-empresa se encuentran: proveedores, competidores, empresas del PADES, otras empresas. En cuanto a cooperación empresas-instituciones, están los gremios y asociaciones empresariales, las cámaras de comercio, los centros y grupos de investigación, las universidades, otros institutos de enseñanza superior y centros de formación profesional, el SENA, las incubadoras de empresas, los centros de desarrollo tecnológico (CDT), Colciencias, los ministerios y las secretarias locales y departamentales.

De igual manera, se logran identificar las principales actividades de cooperación en innovación que realizan las pymes con los agentes anteriormente mencionados. Entre las que se encuentran: tareas conjuntas de $\mathrm{I}+\mathrm{D}$, acuerdos para la capacitación de personal, diseño industrial, tareas de ingeniería, proyectos conjuntos de consultorías, adquisición de equipos, maquinaria y bienes de capital, adquisición de hardware o software, transferencia de tecnología, mejoras asociadas a la organización, compartir información técnica o tecnológica, y acciones como compartir información comercial, realizar alianzas estratégicas para actividades de marketing / comercialización y acuerdos para desarrollo de productos, procesos, tecnologías (OCDE, 2005).

Así mismo, considerando que las universidades tiene potencial para desarrollar $\mathrm{I}+\mathrm{D}+\mathrm{i}$, resulta de especial interés identificar los mecanismos de cooperación que utilizan las pymes con estas. Al respecto, en un estudio para España se encontró que en materia de cooperación universidad-empresa hay un predominio de ciertos tipos de actividades como los proyectos de investigación conjuntos o contratados (Fernández et al., 2011), sumados a otro tipo de actividades como prestar asesoramiento técnico o tecnológico, intercambio de personal y explotación comercial conjunta de patentes e investigaciones.

\subsection{Motivos y obstáculos para la cooperación en innovación}

Para el estudio de la cooperación en innovación es importante, además, el reconocimiento de los motivos que tienen las empresas para participar en este tipo de acuerdos y los factores que los obstaculizan, puesto que pueden servir para proponer estrategias que conduzcan a su implementación y mejoramiento. En relación con los motivos que llevan a las empresas a cooperar en materia de innovación, teorías como la de los recursos, la organización industrial, la dirección estratégica y la gestión, sirven de sustento para su identificación. Éstas enfatizan que los motivos para cooperar residen en la posibilidad de adquirir o mejorar las competencias y capacidades, incrementar la eficiencia, la sinergia y el poder mediante redes y en las limitaciones de recursos de las empresas. No obstante, debido a que pueden ser muy variados, las diferentes perspectivas para analizarlos pueden ser tratadas como complementarias y no como sustitutivas (Bayona, García \& Huerta, 2003).

Para el presente trabajo, basados en la literatura (Bayona et al., 2003; Edwards, Anlló, Castro \& Fernández, 2010; Navarro, 2002; Arvanitis, 2010; Chun \& Mun, 2010) se consideraron tres categorías. Primero, los motivos relacionados con la búsqueda de mercados y oportunidades tales como, acceder a nuevos mercados, lanzar un nuevo producto, mejorar uno existente, mejorar el proceso productivo, mejorar la cadena de comercialización y distribución, mejoras organizacionales, acceder a un insumo o materia prima y mejorar la posición competitiva. Segundo, los referidos a los requisitos financieros o de costos entre los que se encuentran, reducir los riesgos de la actividad innovadora, compartir los costos de los proyectos de $\mathrm{I}+\mathrm{D}+\mathrm{i}$, reducir el tiempo de desarrollo de productos y acceder a recursos públicos de fomento a la innovación. Y por último, los relacionados con las necesidades de conocimiento de las empresas, como acceder a conocimientos de los socios o partners, adquirir nuevas tecnologías, buscar complementariedades tecnológicas y contrarrestar la complejidad del desarrollo tecnológico.

Ahora bien, una vez las empresas deciden cooperar en innovación, al reconocer los motivos que tienen 
para hacerlo, también resulta pertinente conocer los obstáculos que ellas encuentran para llevar a cabo estas actividades. No hay estudios concluyentes al respecto debido a que las empresas están sometidas a las dinámicas de su entorno particular, pero la falta de recursos económicos suele aparecer como el obstáculo más común. De lo anterior, se infiere que las empresas esperan un mayor acceso a financiación cuando cooperan, a la vez que compartir los costos, reduciendo de esta forma la incertidumbre que implican los procesos de innovación (Albizu et al., 2011; Hidalgo \& Albors 2004; Bayona et al., 2003; Edwards et al., 2010).

De igual modo, se identifican otro tipo de obstáculos (Edwards et al., 2010). Algunos situados dentro del contexto interno de la empresa como rigideces organizativas (actitud del personal y de los gestores respecto al cambio), falta de capacitación para gestionar proyectos de innovación en cooperación, falta de información y conocimientos dentro de la misma empresa. Otros se encuentran situados en el contexto social como la falta de cultura para cooperar y la desconfianza, además, se consideraron otras dificultades de tipo logístico como la falta de comunicación y de tiempo. Este tipo de obstáculos plantea riesgos para los acuerdos de cooperación, puesto que favorecen las asimetrías y se convierten en un aspecto negativo a superar debido a que la globalización y la internacionalización de los procesos de $\mathrm{I}+\mathrm{D}+\mathrm{i}$ ofrecen nuevas oportunidades para el establecimiento de redes de conocimiento que las empresas pueden aprovechar para incrementar su competitividad.

\subsection{Resultados de la cooperación en innovación}

Ahora bien, tras exponer los aspectos más importantes en cuanto a la cooperación en innovación se identifican entonces los resultados que obtienen las empresas como producto del desarrollo de diversas actividades que inciden en la realización de innovaciones, lo cual es llamado resultado tecnológico y es medido mediante los objetivos que se pretende conseguir con cada uno de los tipos de innovación considerados: producto, proceso, mercadotecnia y organizativa. La medición de estos resultados de innovación constituye una de las perspectivas mi- croeconómicas para reconocer los efectos que tiene en la empresa la implementación de estrategias para innovar.

De acuerdo con lo anterior, se plantean para cada uno de los cuatro tipos de innovación los siguientes indicadores de resultados (Edwards et al., 2010). Para las innovaciones de producto se consideran las mejoras en los productos de la empresa, introducción de nuevo producto, patente de nuevo producto; para las innovaciones de mercado, mantenimiento, apertura o ampliación de nuevos mercados. En cuanto a innovaciones de proceso se considera, el aumento en capacidad productiva, aumento en la flexibilidad de la producción, reducción de costos, de consumo de materias primas, de consumo de energía, mejoras en aprovechamiento de las competencias de los empleados y patente de un nuevo proceso. De la misma forma, para las innovaciones organizativas se considera la introducción de nuevas prácticas empresariales para organizar rutinas, procedimientos, formas de relación con otras empresas o instituciones y la introducción de métodos atribución de responsabilidades (OCDE, 2005).

\section{Metodología}

Dada la existencia de una abundante pero fragmentada literatura y la carencia de datos secundarios y de referentes teóricos explicativos de la cooperación en innovación, se optó por construir y aplicar una encuesta (Tabla 2 en Anexos) a un universo de empresas susceptibles de haber participado en proyectos de cooperación en innovación, la cual fue diseñada mediante la revisión de diferentes referentes teóricos y metodológicos que sirvieron para la identificación de las principales variables de la cooperación en innovación empresarial.

La unidad de observación son las empresas activas en procesos de cooperación empresarial que hacen parte del Programa de Asociatividad y Desarrollo Empresarial Sectorial -PADES- de la Asociación Colombiana de Micro, Pequeñas y Medianas Empresas -ACOPI-. El instrumento se envió a 60 empresas en el Área Metropolitana del Valle de Aburrá, y se obtuvo respuesta de 19, pertenecientes a los PADES de los sectores salud, artes gráficas, energía, transporte, 
laboratorios, comercio, turismo, hospitalario, litográfico y confecciones.

Las encuestas fueron respondidas en su gran mayoría por gerentes, y por personas de la alta dirección. La información fue recogida correo electrónico, encuesta telefónica y presencial. Dado el carácter exploratorio del estudio y las limitaciones en tamaño de la muestra, el análisis de la información obtenida, se realizó mediante la construcción de tablas de frecuencia y gráficas en excel, que arrojaron los resultados presentados en el siguiente apartado.

\section{Resultados}

A continuación se presentan los resultados de la investigación, los cuales aparecen en el mismo orden que están organizados los ítems en la encuesta. En primer lugar se encuentran, las actividades de innovación realizadas en cooperación, seguido de los agentes con los que se realizó dicha cooperación y la regularidad con la que se hizo; posteriormente están los motivos que consideraron las empresas, los obstáculos que encuentran para desarrollar innovaciones cooperando, los resultados obtenidos para cada tipo de innovación y las fuentes de financiación utilizadas.

\subsection{Actividades de cooperación en innovación}

Al indagar acerca de las actividades de innovación realizadas en cooperación, se encuentra que en primer lugar las empresas cooperan en capacitación del personal con un porcentaje del $78,94 \%$, un $75 \%$ realizó intercambio de conocimientos y tecnología y un $73,68 \%$ ha realizado mejoras en la comercialización. Del mismo modo, las empresas se inclinaron en menor medida hacia algunas actividades como diseño industrial, en un $31,57 \%$, y el $42,1 \%$ de ellas realizó otras actividades tales como, realización de tareas conjuntas de investigación, contratación de servicios $\mathrm{I}+\mathrm{D}$ a terceros, tareas conjuntas de ingeniería, intercambio de personal científico técnico, alquiler de instalaciones o equipos y explotación de alguna patente o desarrollo tecnológico (Gráfica 1).

Gráfica 1. Principales actividades de cooperación en innovación realizadas por las empresas²

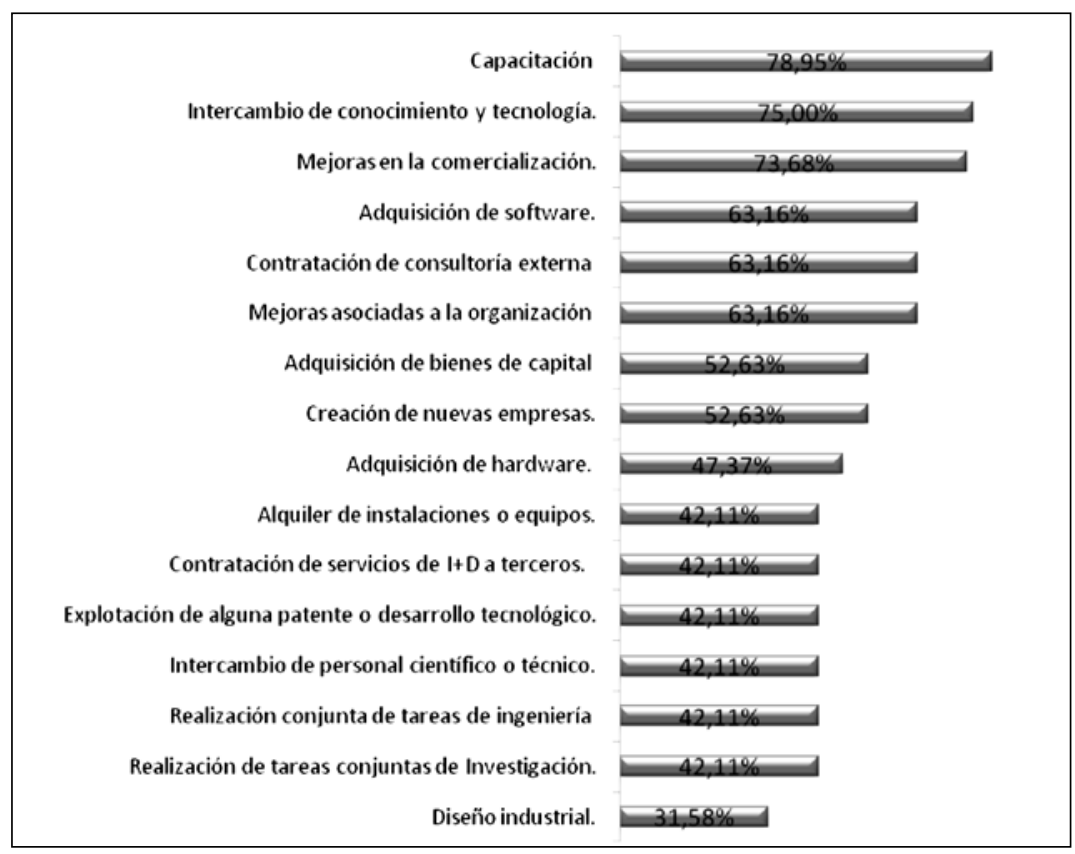

2 Fuente: Elaboración propia. 
Se observa que algunas de las actividades menos intensivas en conocimiento son realizadas más frecuentemente en detrimento de otras con mayor complejidad tales como, realización de tareas conjuntas de investigación, contratación de $\mathrm{I}+\mathrm{D}$ a terceros y realización de tareas conjuntas de ingeniería.

\subsection{Agentes para la cooperación en innovación}

Para este ítem, en promedio $48,11 \%$ del total de empresas indica haber cooperado con alguno de los agentes mencionados, en los últimos tres años. Se observa que las empresas encuestadas, han cooperado en mayor proporción con otras empresas PADES $(84,2 \%)$ en la realización de alguna de las actividades de cooperación en innovación consideradas, seguido por la cooperación con otros gremios y asociaciones empresariales, con un $68,4 \%$. En contraste, han cooperado en menor porcentaje con algunos de los principales actores del Sistema Nacional de Ciencia Tecnología e Innovación, como lo son los centros y grupos de investigación, Colciencias, Centros de Desarrollo
Tecnológico e incubadoras de empresas; menos del $36,8 \%$ de las empresas ha cooperado con alguno de ellos en proyectos de innovación (Gráfica 2).

Lo anterior indica que las empresas de la muestra tienen una clara preferencia por cooperar con otras empresas que hacen parte del entorno PADES, sin embargo, no muestran una inclinación por hacerlo con otros agentes considerados de mayor relevancia para la realización de actividades de $\mathrm{I}+\mathrm{D}+\mathrm{i}$, lo cual va en línea con la baja realización de actividades intensivas en conocimientos.

\subsection{Regularidad de la cooperación}

En lo relativo a la regularidad de la cooperación en innovación, a pesar de que la gran mayoría de las empresas $(73,7 \%)$, indica haber cooperado en proyectos de innovación al menos una vez, alrededor del $47,4 \%$ lo ha hecho en máximo dos ocasiones. Es decir, para un gran porcentaje de las empresas que hacen parte de la muestra no existe un dinamismo significativo en ésta regularidad, puesto que no tienden a establecer relaciones duraderas en las que se coopere para desarrollar proyectos de innovación.

Gráfica 2. Agentes para la cooperación en innovación ${ }^{3}$

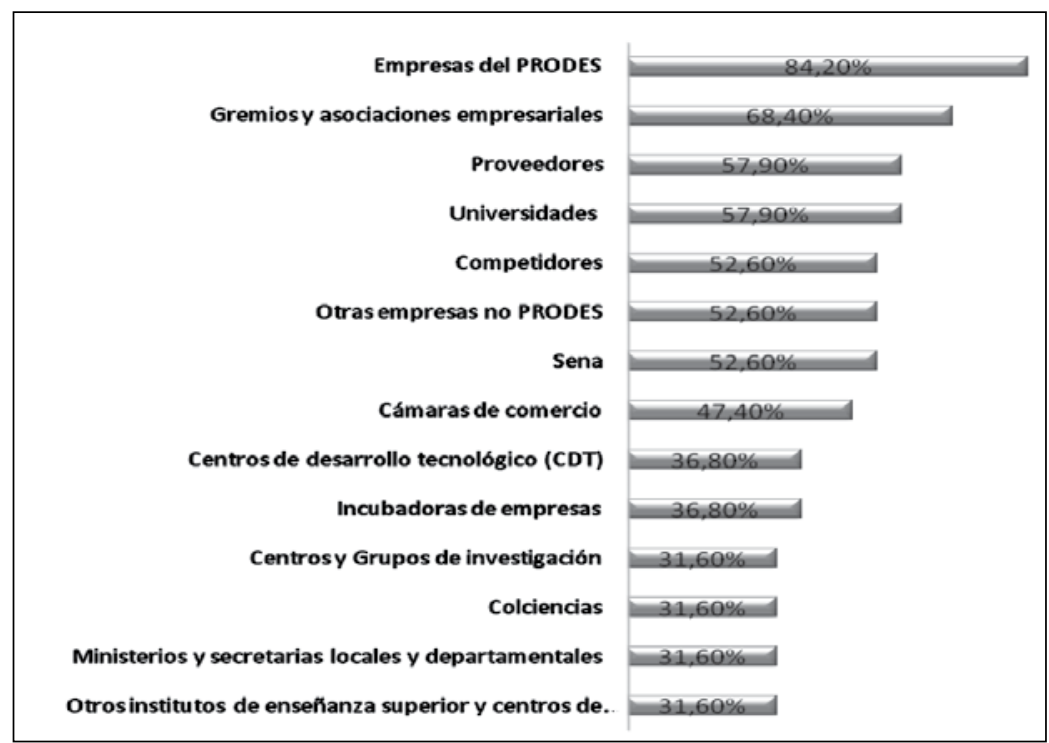

3 Fuente: Elaboración propia. 
Tabla 1. Regularidad en la cooperación en proyectos de innovación ${ }^{4}$

\begin{tabular}{|c|c|c|}
\cline { 2 - 3 } \multicolumn{1}{c|}{} & Frecuencia & $\%$ \\
\hline \multirow{4}{*}{$\begin{array}{c}\text { Regularidad de la } \\
\text { cooperación }\end{array}$} & Nunca & $26,3 \%$ \\
\cline { 2 - 3 } & Una vez & $26,3 \%$ \\
\cline { 2 - 3 } & Dos veces & $21,1 \%$ \\
\cline { 2 - 3 } & Tres veces & $10,5 \%$ \\
\cline { 2 - 3 } & Cuatro veces & $0,0 \%$ \\
\cline { 2 - 3 } & Más de cuatro veces & $15,8 \%$ \\
\cline { 2 - 3 } & Total & $100,0 \%$ \\
\hline
\end{tabular}

\subsection{Motivos para cooperar en innovación}

En la Gráfica 3 se observa que el promedio 53,3\% de las empresas de la muestra indica haber tenido en cuenta alguno de los motivos considerados para hacer innovaciones cooperando con otros agentes. Mejorar la posición competitiva y acceder a nuevos mercados fueron los más comunes con porcentajes del 73,68. En contraste, reducir el tiempo de desarrollo de productos y compartir los costos de los proyectos de $\mathrm{I}+\mathrm{D}+\mathrm{i}$, fueron los menos tomados en cuenta $(36,84 \%)$.

En otras palabras, las razones expuestas por las empresas están más relacionadas con su posición en el mercado y menos orientadas hacia otros motivos mencionados más frecuentemente por la literatura, entre ellos los relativos a las necesidades de conocimiento como reducir la incertidumbre de los procesos de innovación, contrarrestar la complejidad del desarrollo tecnológico y acceder a conocimiento de los socios o partners y los motivos relacionados con la financiación y los costos de las actividades innovadoras.

\subsection{Obstáculos para cooperar en innovación}

Las empresas en conjunto consideran como los obstáculos más importantes, haciendo valoraciones

Gráfica 3. Motivos que consideran las empresas para cooperar en innovación ${ }^{5}$

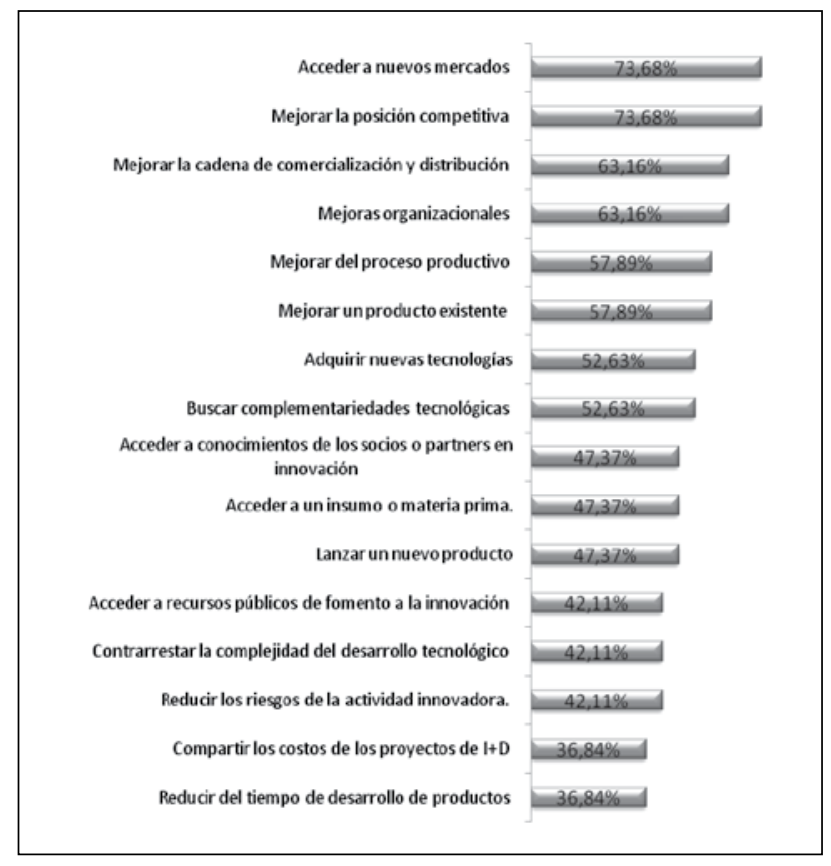

4 Fuente: Elaboración propia.

5 Fuente: Elaboración propia. 
altas para ellos, los siguientes aspectos: falta de cultura para cooperar $(3,10)$, falta de confianza $(3,21)$, falta de recursos económicos $(3,10)$ y falta de capacitación para gestionar proyectos de innovación en cooperación $(3,10)$. En contraste, valoran la falta de conocimientos del agente con el cual se cooperó como el obstáculo con menor importancia $(1,84)$ (Gráfica 4).

De acuerdo con lo anterior, las empresas encuentran principalmente en factores externos relacionados con la cultura empresarial local, los mayores impedimentos para cooperar en innovación, lo cual puede ser un reflejo de los bajos indicadores de cooperación con otros agentes fuera del entorno PADES. En segundo lugar, las dificultades relacionadas con la falta de recursos económicos y conocimientos, podrían estar explicando la mayor frecuencia en el desarrollo de actividades de menor complejidad, como lo indican los resultados en el apartado de actividades.

\subsection{Resultados en innovación}

En este apartado se presentan los resultados obtenidos por las empresas para cada tipo de innovación
(Gráfica 5); de producto, marketing, proceso y organizacional. En promedio un 73,66\% de las empresas indica haber obtenido resultados en innovaciones de marketing, lo que es coherente con las razones que expresan para cooperar en innovación, las cuales están más relacionadas con su posición en el mercado, más exactamente, las empresas ampliaron su cuota de mercado $(84,1 \%)$ y abrieron nuevos mercados (78,95\%). En segundo lugar, un $66,7 \%$ indica haber realizado innovaciones organizativas, $73,68 \%$ introdujo nuevas maneras de relacionarse con otras empresas o instituciones, en los últimos tres años; esto va en línea con la participación de las empresas en actividades conjuntas en el programa PADES, que implica otras formas de relacionarse con diferentes empresas o instituciones en la búsqueda de objetivos comunes.

Adicionalmente, los resultados muestran que en promedio $57,89 \%$ de las empresas obtuvo algún resultado en innovación de producto, el 73,68\% mejoró los productos de la empresa o introdujo un nuevo producto $(63,16 \%)$. Por el lado de las innovaciones de proceso, un promedio de $43,61 \%$ de empresas obtuvo algún resultado de este tipo, cabe destacar

Gráfica 4. Valoración promedio de todas las empresas para los obstáculos para cooperar en innovación ${ }^{6}$

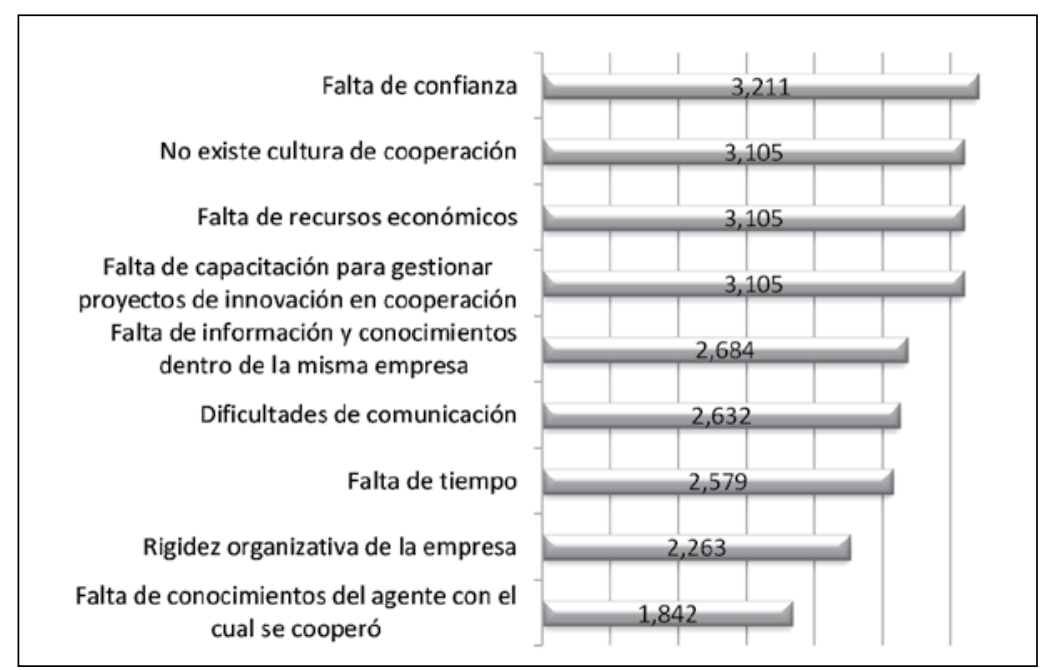

6 Fuente: Elaboración propia. 
Gráfica 5. Resultados obtenidos por las empresas en los cuatro tipos de innovación durante el período de análisis 2009-2012

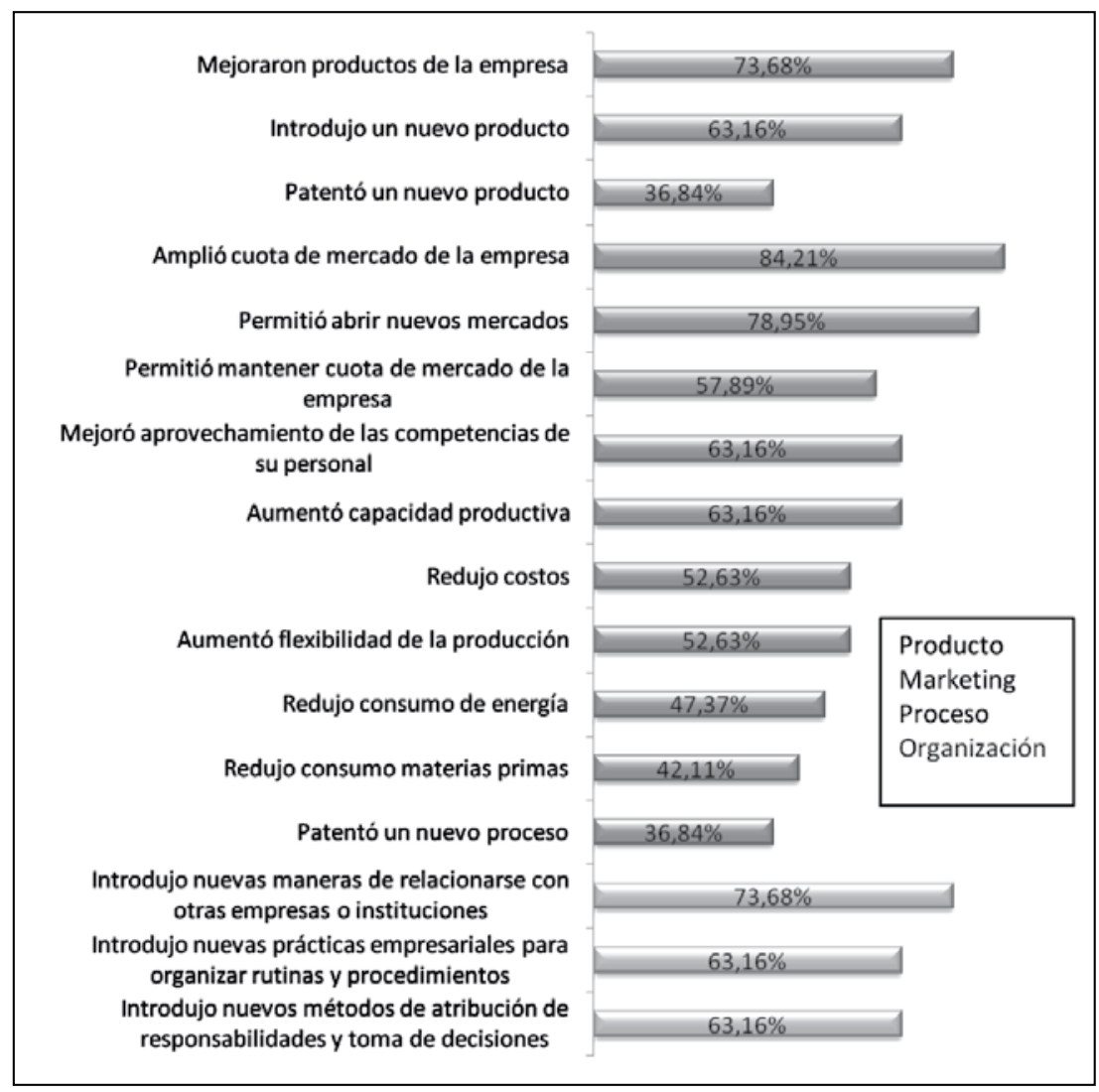

que estas innovaciones fueron las menos comunes entre los cuatro tipos; en mayor medida, las empresas aumentaron su capacidad productiva $(63,16 \%)$, o mejoraron el aprovechamiento de las capacidades de su personal $(63,16 \%)$.

\subsection{Financiación}

En las Mipymes suele existir un déficit de fondos propios y un menor acceso a recursos del sistema financiero para realizar proyectos de innovación, pero los que realizan, en su mayoría son financiados con recursos de su propiedad como lo indican los resultados obtenidos en este apartado el cual se considera relevante en la medida en que permite tener una primera idea sobre las dificultades financieras que podrían afectar la capacidad de las empresas encuestadas para innovar.

Los resultados en cuanto a las fuentes de financiación para proyectos de innovación en el período 2009-2012 (Gráfica 6), muestran que $89,5 \%$ de las empresas utilizaron recursos propios para financiar el $50 \%$ o más de los proyectos de innovación que emprendieron en el período de análisis y en segundo lugar, el 68,4\% utilizó recursos de la banca privada, a su vez, el $52,6 \%$ y el $47,4 \%$ indica haber utilizado en alguna medida recursos pertenecientes a otras empresas PADES y recursos públicos respectivamente, por último, sólo un $21,1 \%$ utilizó alguna vez recursos de donaciones.

\footnotetext{
7 Fuente: Elaboración propia.
} 
Gráfica 6. Fuentes de financiación ${ }^{8}$

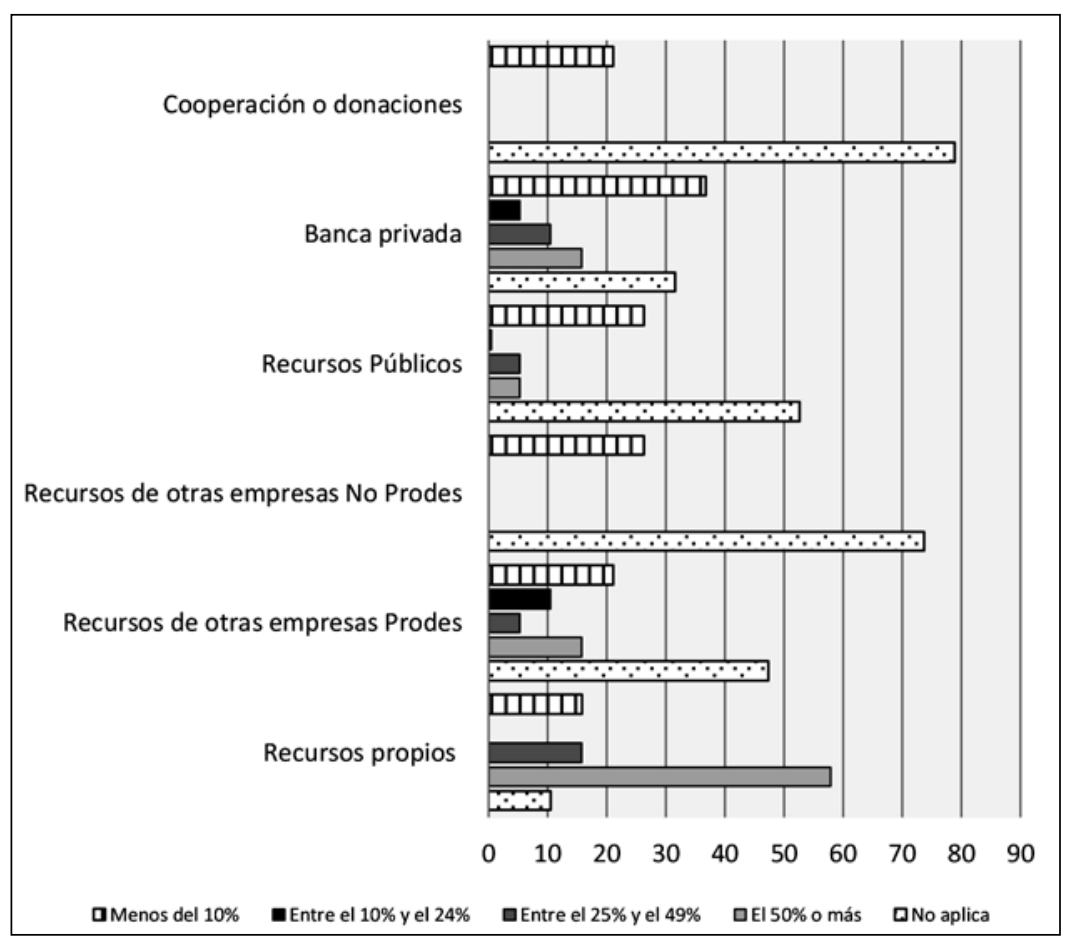

\section{Conclusiones}

Los resultados muestran que poco más de la mitad de las empresas indica no haber realizado ninguna actividad de cooperación en innovación, es decir, gran parte de las empresas encuestadas implementan los mecanismos de cooperación del PADES a través de la asociatividad, pero fruto de esta estrategia no realizan actividades conjuntas para innovar y en las que lo hacen, se observa que tienden a cooperar más en actividades de intercambio simple de conocimiento y tecnologías, capacitación de personal y mejoras en la comercialización, lo que lleva a pensar que existe un predominio de intercambio de conocimiento tácito $y$ de experiencias adquiridas.

Se destaca que las empresas tienden a cooperar más con otras empresas PADES y con gremios o asociaciones empresariales, pero lo hacen en menor medida con agentes generadores de conocimiento más especializado como centros y grupos de investigación, además de otros agentes que hacen parte fundamental de las políticas de Ciencia, Tecnología e Innovación como Centros de Desarrollo Tecnológico, Colciencias e incubadoras de empresas. Dado que las empresas de la muestra no suelen realizar actividades de cooperación en innovación que conlleven intercambio de conocimiento complejo, es de esperar que los intercambios de información y conocimiento simple que realizan sean en su mayoría con empresas con las que interactúan dentro del mismo programa.

Las empresas encuestadas en mayor porcentaje reconocen como motivos para realizar innovaciones cooperando, los relacionados con el mercado, como por ejemplo mejorar su posición competitiva, y acceder a nuevos mercados; y le otorgan una menor importancia a los relacionados con los costos y la incertidumbre de la innovación. Es decir, las empresas de la muestra optan más frecuentemente por coo-

8 Fuente: Elaboración propia. 
perar para desarrollar innovaciones de marketing y organizacionales, en las cuales se muestran los mayores resultados en el período de análisis, este hecho puede estar ligado igualmente con las mejoras en la comercialización; una de las actividades de cooperación en innovación que más realizan.

En cuanto a los obstáculos que señalan las empresas para cooperar en innovación, son de mayor importancia la falta de cultura de cooperación y la falta de confianza, los cuales son compatibles con la cultura empresarial tradicional de las empresas colombianas, en la que las empresas tienen reservas a la hora de cooperar con otros agentes y tienden a adoptar posiciones aislacionistas. Aunque fueron considerados también la falta de recursos económicos y la falta de conocimiento para gestionar proyectos de innovación, comúnmente mencionados en la literatura.

El poco dinamismo en la regularidad de la cooperación puede estar relacionado, en primer lugar, con los principales obstáculos que tienen las empresas para cooperar y en segundo lugar, con los tipos de innovación obtenidos con mayor frecuencia (marketing y organizacional), los cuales no requieren de horizontes de investigación más amplios y conocimientos complejos como lo pueden requerir las innovaciones de proceso y producto.

Si se comparan las innovaciones realizadas por las empresas, con el interés de la política pública se encuentra que existe una divergencia. Las innovaciones realizadas tienden a ser de carácter blando, con poco valor agregado, dado que además los empresarios tienden a confundir la innovación con la adopción de nuevas tecnologías. Este hecho es contrario al deseo de la Política Nacional de Ciencia Tecnología e Innovación, la cual busca promover las actividades de Investigación y Desarrollo, y la generación de conocimiento. En este sentido, se hace necesario encontrar mecanismos para fortalecer y mejorar los procesos de innovación que realizan más comúnmente las empresas y en particular las Pymes, los cuales, pueden constituir una base para el logro de innovaciones más complejas.

Es pertinente realizar seguimiento y evaluación exhaustiva de las innovaciones que obtienen las empresas pertenecientes a programas estratégicos como el PADES, con el propósito de conocer más a fondo el impacto que tienen los procesos asociativos para la consecución de innovaciones y las acciones de política pública que se puedan implementar para lograr mejores resultados en este campo. Dentro de las cuales se pueden considerar el darle una mejor relevancia a los centros especializados en innovaciones blandas de organización y marketing que se puedan configurar en la financiación de éste tipo de proyectos.

Esta primera aproximación es de un nivel exploratorio, debido a la poca investigación sobre el tema objeto de estudio y las limitaciones muestrales, que pretende establecer un punto de partida para investigaciones futuras y abrir un camino para mejorar $e$ implementar el instrumento metodológico desarrollado. En este sentido, se sugieren nuevas investigaciones que permitan explicar algunos resultados importantes encontrados, como el hecho de que la cooperación gira en torno a la innovación en marketing y no se extiende hacia la de producto y otras de mayor impacto y complejidad.

\section{Referencias}

Albizu, E., Olazaran, M., Otero, B. \& Lavía, C. (2011). Innovación en las pymes industriales: una visión desde el modelo interactivo. En: Revista Internacional de Organizaciones, 7: 17-43.

Aldrich, H. (1976). Resource dependence and interorganizational relations: relations between local employment service offices and social service sector organizations. En: Administration and Society, 7: 419-454.

Aldrich, H. \& Pfeffer, J. (1976). Environments of organizations. En: Annual Review of Sociology, 2: 79-105.

Alm, H. \& McKelvey, M. (2000). When and why does cooperation positively or negatively affect innovation? An exploration into turbulent waters. Centre for Research on Innovation and Competition, University of Manchester. CRIC Discussion Paper No39. Disponible en: http://www.cric.ac.uk/cric/Pdfs/dp39.pdf

Arvanitis, S. (2010). How do different motives for R\&D cooperation affect firm performance? An analysis based on Swiss micro data. Imperial College London Business School. Disponible en: http:// www.kof.ethz.ch/en/publications/p/kof-working-papers/233/

Barney, J. (1991). Firm resources and sustained competitive advantage. En: Journal of Management, 17(1): 99-120.

Barney, J. \& Arikan, A. (2001). The resource-based view: origins and implications. The Blackwell Handbook of Strategic Management, Oxford.

Bayona, C., García, T. \& Huerta, E. (2003). ¿Cooperar en I+D? Con quién y para qué?. En: Revista de Economía Aplicada EA., 11( 31): 103-134. 
Becker, W. \& Dietz, J. (2004). R\&D co-operation and innovation activities of firms-evidence for German manufacturing industry. En: Research Policy, 33(2): 209-223.

Boltri, P. (2006). Redes de innovación y cooperación: un análisis del sector informático de la ciudad de Mar del Plata. Tesis de Grado Licenciatura en Economía, Universidad Nacional de Mar del Plata, Mar del Plata, Argentina. Disponible en: http:// nulan.mdp.edu.ar/619/1/boltri p.pdf

Cataño, G., Botero, P. \& Vanegas, J. (2008). Redes de conocimiento en sistemas regionales de innovación: un estudio comparado, el caso de las pymes en Antioquia y el País Vasco. Medellín: Instituto Técnico Metropolitano (ITM) y Cámara de Comercio de Medellín para Antioquia.

Chun, H. \& Mun, S. (2010). Determinants of R\&D cooperation in small and medium-sized enterprises. En: Small Business Economics, 39(2): 419-436. Disponible en: http://hompi.sogang. ac.kr/hchun/chun sbe forthcoming.pdf

Cohen, W. \& Levinthal, D. (1989). Innovation and learning: the two faces of R\&D. En: The Economic Journal, 99: 569-596.

Cohen, W. \& Levinthal, D. (1990). Absorptive capacity: a new perspective on learning and motivation. En: Administrative Science Quarterly, 35: 128-152.

Consejo Nacional de Política Económica y Social -CONPES (2007). Política nacional para la transformación productiva y la promoción de las micro, pequeñas y medianas empresas. Un esfuerzo público privado. Documento CONPES 3484. Bogotá. Disponible en: http://www.huila.gov.co/documentos/C/ CONPES3484de2007.pdf

Departamento Administrativo Nacional de Estadística -DANE (2005). Censo General 2005. Disponible en: https://www.dnp. gov.co/LinkClick.aspx?fileticket $=$ aGltR-nsPrU\%3d\&tabid $=809$

Edwards, M., Anlló, G., Castro, E. \& Fernández, I. (2010). Cooperación en I+D e innovación entre empresas argentinas y españolas: una aproximación empírica. En: Revista Iberoamericana de Ciencia, Tecnología y Sociedad, 6(16): 91-121.

Fernández, M., Merchán, C., Rodríguez, L. \& Valmaseda, O. (2011). Indicadores de transferencia de conocimiento: una propuesta de medida de la cooperación entre universidad y empresa. Instituto de Estudios Sociales Avanzados, Consejo Superior de Investigaciones Científicas. Córdoba, España. Disponible en: http://congreso.ricyt.org/files/Indic_\%20CTI/Indicadores\%20 de\%20transferencia\%20de\%20conocimiento.pdf

Filiou, D. \& Massini, S. (2009). Cooperation and innovation: the role of alliance capability creation. Copenhagen Business School. Disponible en: http://www2.druid.dk/conferences/viewpaper. php?id $=5734 \& \mathrm{cf}=32$

Giraldo, A., Bedoya, G. \& Vargas, C. (2009). Principales limitaciones del empresarismo que afectan el desarrollo económico y social del país. En: Revista: Escuela de Administración de Negocios - EAN, (66): 99-112.

Godoe, H. (2000). Innovation regimes, R\&D and radical innovations in telecommunications. En: Research Policy, 29(9): 1033-1046.

Hidalgo, A. \& Albors, J. (2004). La internacionalización de la tecnología a través de los proyectos de IBEROEKA. En: Cuadernos de Economía y Dirección de la Empresa, (20): 57-82.

Jaklič, A., Domiján, J. \& Rojec, M. (2010). Innovation cooperation and innovation activity: the impact on the case of Slovenian enterprises. Disponible en: https://iweb.cerge-ei.cz/pdf/gdn/ RRCVII_52_paper_01.pdf
Jaramillo, H., Lugones, G. \& Salazar M., (2001). Normalización de indicadores de innovación tecnológica en América Latina y el Caribe. Manual de Bogotá. RICYT, OEA y OCYT. Disponible en: http://www.uis.unesco.org/Library/Documents/Bogota\%20 Manual_Spa.pdf

Jardón, C. (2011). Innovación empresarial y territorio: una aplicación a Vigo y su área de influencia. En: EURE - Santiago, 37(112): 115-139.

Lavía, C., Otero, B., Olazaran, M. \& Albizu, E. (2011). Innovación y territorio: una encuesta a pequeñas y medianas empresas industriales. En: Revista Internacional de Sociología (RIS), 69(2): 461-486.

López, A. (2008). Cooperation in innovative activities, organizational innovation and productivity: three essays on economics of innovation. Tesis de doctorado Universidad Complutense de Madrid, España. Disponible en: http://eprints.ucm.es/8829/1/ T30831.pdf.

Lundvall, B. (1992). National system of innovation. Towards a theory of innovation and interactive learning. Londres, Pinter Publishers.

Malaver, F. \& Vargas, M. (2006). Capacidades tecnológicas, innovación y competitividad de la industria de Bogotá y Cundinamarca, resultados de una encuesta de innovación. Cámara de Comercio de Bogotá y Observatorio Colombiano de Ciencia y Tecnología. Disponible en: http://ocyt.org.co/html/archivosProyectos/EIByC.pdf

Monroy, S. (2006). Nuevas políticas y estrategias de articulación del sistema de ciencia, tecnología e innovación colombiano. En: Revista INNOVAR, 16(28): 157 -171.

Muñoz, J. \& Montoro, M. (2006). Enfoques teóricos para el estudio de la cooperación empresarial. En: Cuadernos de estudios empresariales, (17): 141-163.

Navarro, M. (2002). La cooperación para la innovación en la empresa española desde una perspectiva internacional comparada. En: Revista de economía industrial, 4(346): 47-66.

Organización para la Cooperación y el Desarrollo Económicos-OCDE (2005). Manual de Oslo. Guía para la recogida e interpretación de datos sobre innovación. Tercera edición. Disponible en: www.conacyt.gob.sv/Indicadores\%20Sector\%20Academcio/ Manual de Oslo\%2005.pdf

Pallares, Z. (2000). La Asociatividad Empresarial, una respuesta de los pequeños productores a la internacionalización de las economías. Programa de Desarrollo Empresarial Sectorial PRODES. Disponible en: http://www.cadenahortofruticola.org/ admin/geren/60internacionalizacion_economia.pdf

Pfeffer, J. \& Salancik, G. (1978). The external control of organizations: a resource dependence perspective. Stanford business classics, Stanford, California.

Porter, M. (1991). Towards a dynamic theory of strategy. En: Strategic Management Journal, 12: 95-117.

Powell, W. \& Grodal, S. (2005). Networks of innovators. The Oxford Handbook of Innovation, Oxford University Press.

Rojec, M. \& Jaklič, A. (2002). Integration of Slovenia into EU and global industrial networks: review of existing evidence. Economics Working Papers $\mathrm{N}^{\circ} 14$. Centre for the Study of Economic and Social Change in Europe, SSEES, UCL. Disponible en: http:// discovery.ucl.ac.uk/17565/

Rosales, R. (1997). La Asociatividad como estrategia de fortalecimiento de las pymes. Universidad de Texas. Disponible 
en: http://www.sela.org/attach/258/EDOCS/SRed/2005/11/ T023600000022-7-La_asociatividad_como_estrategia.htm Schmalensee, R. \& Willig, $\bar{R}$. (1989). Hañdbook of Industrial Organization. North Holland.

Schumpeter, J. (1935). Analysis of economic change. En: The Review of Economics and Statistics, Massachusetts Institute of Technology, MIT Press, 17(4): 2-10.

Tirole, J. (1988). The theory of industrial organization. Massachusetts Institute of Technology, MIT Press. Disponible en: https://faculty.fuqua.duke.edu/ charlesw/s591/willstuff/oldstuff/ PhD_2007-2008/Papers/c06/Tirole\%20\%28Part\%202\%29\%20 Class\%206.pdf

Wernerfelt, B. (1984). A resource-based view of the firm. En: Strategic Management Journal, 5(2): 171-180.

Williamson, O. (1979). Transaction-cost economics: the governance of contractual relations. En: Journal of Law and Economics, 22 (2). 


\section{ANEXOS}

Tabla 2. Encuesta ${ }^{1}$

\begin{tabular}{|l|l|}
\hline \multicolumn{2}{|c|}{ Encuesta innovación en cooperación en empresas } \\
\hline Nombre de la empresa: & \\
\hline Número de empleados: & \\
\hline Año de creación de la empresa: & \\
\hline Año de pertenencia al PADES: & \\
\hline Nombre del encuestado: & \\
\hline Cargo del encuestado: & \\
\hline Tipo de empresa & \\
\hline
\end{tabular}

1. Indique cuáles actividades ha realizado cooperando con otras empresas y/o agentes en los últimos tres años y señale con una X su grado de importancia para el desarrollo de innovaciones. Para aquellas actividades que usted no realizó marque con X la opción NO APLICA.

\begin{tabular}{|c|c|c|c|c|c|c|}
\hline \multirow[b]{2}{*}{ Actividades de cooperación en innovación } & \multicolumn{5}{|c|}{ Grado de importancia } & \multirow{2}{*}{$\begin{array}{c}\text { No } \\
\text { aplica }\end{array}$} \\
\hline & $\begin{array}{l}\text { Muy } \\
\text { Baja }\end{array}$ & Baja & Media & Alta & Muy Alta & \\
\hline \multicolumn{7}{|l|}{ Realización de tareas conjuntas de Investigación } \\
\hline \multicolumn{7}{|l|}{ Contratación de servicios de I+D a terceros } \\
\hline \multicolumn{7}{|l|}{ Intercambio de conocimiento y tecnología } \\
\hline \multicolumn{7}{|l|}{ Realización conjunta de tareas de ingeniería } \\
\hline \multicolumn{7}{|l|}{ Diseño industrial } \\
\hline \multicolumn{7}{|l|}{ Adquisición de software } \\
\hline \multicolumn{7}{|l|}{ Adquisición de hardware } \\
\hline \multicolumn{7}{|l|}{ Adquisición de bienes de capital } \\
\hline \multicolumn{7}{|l|}{ Capacitación } \\
\hline \multicolumn{7}{|l|}{ Contratación de consultoría externa } \\
\hline \multicolumn{7}{|l|}{ Mejoras asociadas a la organización } \\
\hline \multicolumn{7}{|l|}{ Intercambio de personal científico o técnico } \\
\hline \multicolumn{7}{|l|}{ Alquiler de instalaciones o equipos } \\
\hline \multicolumn{7}{|l|}{ Explotación de alguna patente o desarrollo tecnológico } \\
\hline \multicolumn{7}{|l|}{ Mejoras en la comercialización } \\
\hline \multicolumn{7}{|l|}{ Creación de nuevas empresas } \\
\hline Otros ¿Cuáles? & & & & & & \\
\hline
\end{tabular}

2. Indique con cuáles agentes ha cooperado en la realización de actividades de innovación en los últimos 3 años y señale con una X el grado de importancia del agente para el desarrollo de innovaciones. Para aquellos agentes con los cuales no ha cooperado marque con X la opción NO APLICA.

1 Fuente: Elaboración propia. 


\begin{tabular}{|c|c|c|c|c|c|c|}
\hline \multirow{2}{*}{$\begin{array}{l}\text { Agentes para la cooperación en inno- } \\
\text { vación }\end{array}$} & \multicolumn{5}{|c|}{ Grado de importancia } & \multirow[b]{2}{*}{ No aplica } \\
\hline & $\begin{array}{l}\text { Muy } \\
\text { Baja }\end{array}$ & Baja & Media & Alta & Muy Alta & \\
\hline \multicolumn{7}{|l|}{ Proveedores } \\
\hline \multicolumn{7}{|l|}{ Competidores } \\
\hline \multicolumn{7}{|l|}{ Empresas del PADES } \\
\hline \multicolumn{7}{|l|}{ Otras empresas } \\
\hline \multicolumn{7}{|l|}{ Gremios y asociaciones empresariales } \\
\hline \multicolumn{7}{|l|}{ Cámaras de comercio } \\
\hline \multicolumn{7}{|l|}{ Centros y Grupos de investigación } \\
\hline \multicolumn{7}{|l|}{ Universidades } \\
\hline \multicolumn{7}{|l|}{$\begin{array}{l}\text { Otros institutos de enseñanza superior y centros de } \\
\text { formación profesional }\end{array}$} \\
\hline \multicolumn{7}{|l|}{ Sena } \\
\hline \multicolumn{7}{|l|}{ Incubadoras de empresas } \\
\hline \multicolumn{7}{|l|}{ Centros de desarrollo tecnológico (CDT) } \\
\hline \multicolumn{7}{|l|}{ Colciencias } \\
\hline \multicolumn{7}{|l|}{ Ministerios y secretarias locales y departamentales } \\
\hline Otros ¿Cuáles? & & & & & & \\
\hline
\end{tabular}

3. Indique el número de veces que su empresa ha cooperado en proyectos de investigación, desarrollo $e$ innovación $(\mathrm{I}+\mathrm{D}+\mathrm{i})$ con otras empresas y/o agentes en los últimos 3 años.

\begin{tabular}{|l|l|l|l|l|l|l|}
\hline & Nunca & $\mathbf{1}$ vez & $\mathbf{2}$ veces & 3 veces & $\mathbf{4}$ veces & $\begin{array}{c}\text { Más de } 4 \\
\text { veces }\end{array}$ \\
\hline Regularidad de la cooperación & & & & & & \\
\hline
\end{tabular}

4. Indique cuáles motivos tuvo para realizar actividades de innovación en cooperación con otras empresas y/o agentes y señale con una X su grado de importancia. Para aquellos motivos que usted no tuvo en cuenta marque con X la opción NO APLICA

\begin{tabular}{|l|l|l|l|l|l|l|}
\hline \multirow{2}{*}{ Motivos para cooperar en innovación } & \multicolumn{5}{c|}{ Grado de importancia } & \multirow{2}{*}{ No aplica } \\
\cline { 2 - 7 } & $\begin{array}{c}\text { Muy } \\
\text { Baja }\end{array}$ & Baja & Media & Alta & Muy Alta & \\
\hline Acceder a nuevos mercados & & & & & & \\
\hline Lanzar un nuevo producto & & & & & & \\
\hline Mejorar un producto existente & & & & & & \\
\hline Mejorar el proceso productivo & & & & & & \\
\hline Mejorar la cadena de comercialización y distribución & & & & & & \\
\hline Mejoras organizacionales & & & & & & \\
\hline Acceder a un insumo o materia prima & & & & & & \\
\hline Mejorar la posición competitiva & & & & & & \\
\hline Reducir los riesgos de la actividad innovadora & & & & & & \\
\hline
\end{tabular}




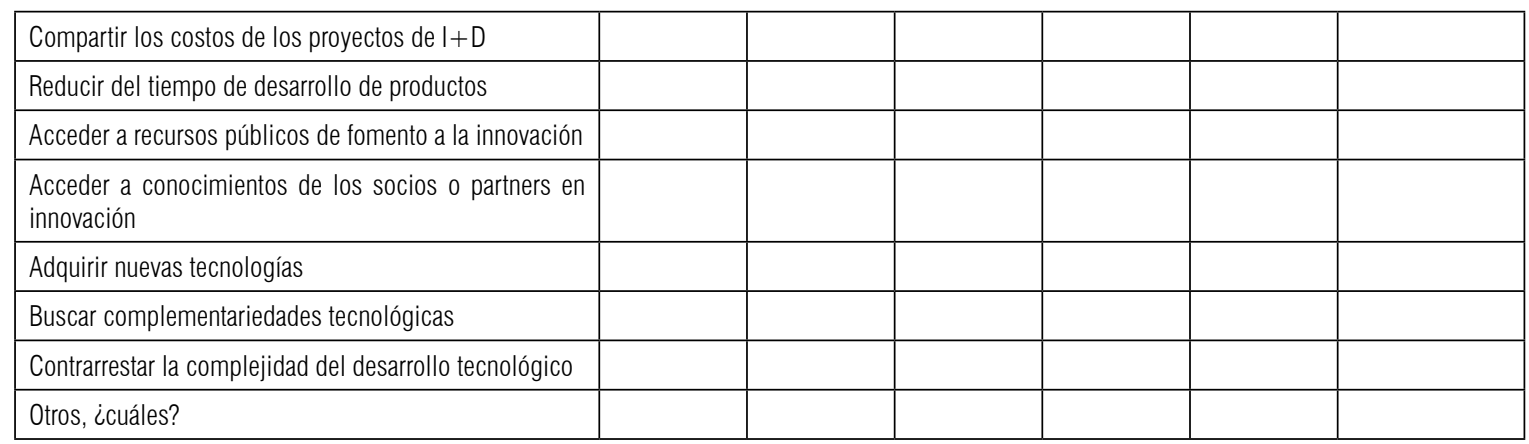

5. Indique cuáles obstáculos ha tenido para realizar actividades de innovación en cooperación con otras empresas $\mathrm{y} / \mathrm{o}$ agentes en los últimos tres años y señale con una $\mathrm{X}$ su grado de incidencia.

\begin{tabular}{|l|l|l|l|l|l|}
\hline \multicolumn{2}{|c|}{ Factores que obstaculizan la cooperación en } \\
innovación & \multicolumn{1}{c|}{ Grado de incidencia } \\
\cline { 2 - 6 } & Muy Baja & Baja & Media & \multicolumn{1}{c|}{ Alta } & Muy Alta \\
\hline No existe cultura de cooperación & & & & & \\
\hline Falta de confianza & & & & & \\
\hline Falta de recursos económicos & & & & & \\
\hline Rigidez organizativa de la empresa & & & & & \\
\hline $\begin{array}{l}\text { Falta de capacitación para gestionar proyectos de innovación en } \\
\text { cooperación }\end{array}$ & & & & & \\
\hline $\begin{array}{l}\text { Falta de información y conocimientos dentro de la misma em- } \\
\text { presa }\end{array}$ & & & & & \\
\hline Falta de conocimientos del agente con el cual se cooperó & & & & & \\
\hline Dificultades de comunicación & & & & & \\
\hline Falta de tiempo & & & & & \\
\hline Otros icuáles? & & & & & \\
\hline
\end{tabular}

6. Indique cuáles resultados obtuvo en materia de innovación en los últimos 3 años y señale con una X el grado de importancia en terminos económicos para su empresa. Para aquellos resultados que usted no obtuvo marque con X la opción NO APLICA.

\begin{tabular}{|l|l|l|l|l|l|l|}
\hline \multicolumn{1}{|c|}{ Resultados } & \multicolumn{3}{c|}{ Grado de importancia } & \multirow{2}{*}{ No aplica } \\
\hline Mejoraron productos de la empresa & Muy Baja & Baja & Media & \multicolumn{1}{c|}{ Alta } & Muy Alta & \\
\hline Introdujo un nuevo producto & & & & & & \\
\hline Patentó un nuevo producto & & & & & & \\
\hline Otro, icuál? & & & & & & \\
\hline Marketing & & & & & & \\
\hline Permitió mantener cuota de mercado de la empresa & & & & & & \\
\hline Amplió cuota de mercado de la empresa & & & & & & \\
\hline Permitió abrir nuevos mercados & & & & & & \\
\hline Otro, icuál? & & & & & & \\
\hline
\end{tabular}




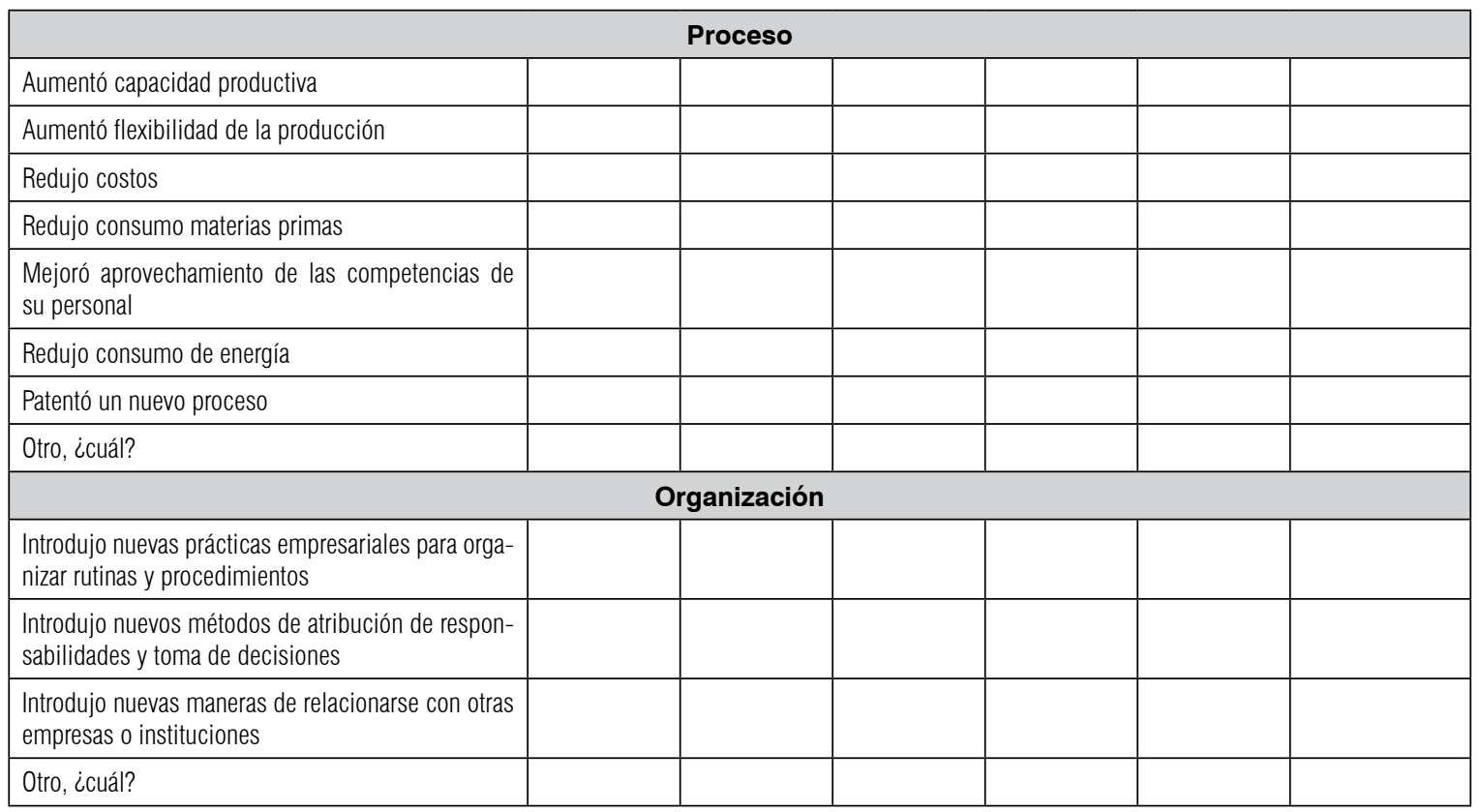

7. Indique cuáles fuentes de financiación ha utilizado en proyectos de innovación llevados a cabo en los últimos tres años y señale el porcentaje de financiación obtenido de cada una. Para aquellas fuentes de financiación no utilizadas seleccione la opción NO APLICA.

\begin{tabular}{|l|l|l|l|l|l|}
\hline \multicolumn{1}{|c|}{$\begin{array}{c}\text { Encuesta innovación } \\
\text { en Cooperación en empresas }\end{array}$} & $\begin{array}{c}\text { Menos del } \\
\mathbf{1 0 \%}\end{array}$ & $\begin{array}{c}\text { Entre el 10\% } \\
\text { y el 24\% }\end{array}$ & $\begin{array}{c}\text { Entre el 25\% } \\
\text { y el 49\% }\end{array}$ & $\begin{array}{c}\text { El 50\% o } \\
\text { más }\end{array}$ & No aplica \\
\hline Recursos propios de la empresa & & & & & \\
\hline Recursos de otras empresas del PADES & & & & & \\
\hline Recursos de otras empresas no PADES & & & & & \\
\hline Recursos públicos & & & & & \\
\hline Recursos de la banca privada & & & & & \\
\hline Recursos de cooperación o donaciones & & & & & \\
\hline Otra fuente, icuál? & & & & & \\
\hline
\end{tabular}

